\title{
TESTE DE PERDA CRÍtICA PARA DELIMITAÇÃO dO MERCADO RELEVANTE: APLICAÇÃO NO MERCADO DE AUTOMÓVEIS
}

\author{
Jônatas Henrique Souza dos Santos' \\ Mariusa Momenti Pitelli²
}

\section{Resumo}

A definição de mercado relevante é importante para a análise de atos de concentração no âmbito da defesa de concorrência, seguindo o exercício do Teste do Monopolista Hipotético (TMH). Para implementar o TMH, o Teste de Perda Crítica é utilizado para mensurar o mercado relevante. Assim, o presente estudo tem como objetivo aplicar para o mercado brasileiro de automóveis o Teste de Perda Crítica, especificamente para os modelos Uno, Gol e Celta no subsegmento Veículos de Entrada. Observou-se que, de forma geral, a delimitação do mercado relevante nas simulações realizadas fica dependente da margem considerada. Nota-se nas simulações, considerando as margens de referência, que o modelo Gol 1.0 é forte candidato em estar no mesmo mercado relevante que o Celta 1.0 e o Uno 1.0.

Palavras-chave: Mercado Relevante; Teste de perda crítica; Poder de mercado; Automóvel; Veículos de Entrada

Classificação JEL: L1; L4

Bacharel em Ciências Econômicas pela Universidade Federal de São Carlos (UFSCar). E-mail: jonataste.henrique@gmail.com

Mestrado e Doutorado em Economia Aplicada pela ESALQ/USP. Professora Adjunta da Universidade Federal de São Carlos (UFSCar). E-mail: mariusa@ufscar.br 


\title{
1. INTRODUÇÃO
}

Em uma análise de atos de concentração, um dos pontos mais importantes para avaliar o poder de mercado é a definição do mercado relevante. Segundo Possas (1996):

\begin{abstract}
"um mercado só é relevante para a análise de efeitos anticompetitivos potenciais se for um espaço econômico (definido em termos geográficos e de produto) no qual algum poder de mercado tenha possibilidade a priori de ser exercido, este último, por sua vez, também pressupõe obviamente uma definição de mercado tal que ele possa ser exercido" (POSSAS, 1996, p.1).
\end{abstract}

Dessa forma, a partir do mercado relevante corretamente delineado é possível verificar se um ato de concentração (fusão, aquisição, incorporação ou joint venture) entre empresas em um determinado mercado trará resultados que prejudiquem a concorrência, como por exemplo, elevação de preços ao consumidor.

A definição do mercado relevante é uma etapa importante para a análise de fusões e aquisições, pois, de acordo com Possas (1996), é nesse mercado que ocorre o exercício de poder de mercado. Está presente nos "Guias de Análise de Atos de Concentração" (Guias) de diversos países - inclusive no Guia brasileiro ${ }^{3}$ - os quais direcionam a análise dos órgãos antitruste sobre a possibilidade do exercício de poder de mercado resultante de atos de concentração realizados em determinados mercados.

Então, tendo como referência as instruções presentes no Guia brasileiro (BRASIL, 2001), a análise dessas operações notificadas aos órgãos antitruste é feita considerando um mercado relevante definido, onde são calculados os índices de concentração que irão nortear a análise da possibilidade do exercício de poder de mercado, e caso haja necessidade, ocorrerá a verificação das condições de entrada e de rivalidade nesse mercado relevante, bem como possíveis eficiências geradas.

Uma das formas encontradas na literatura para se definir o mercado relevante é por meio do Teste de Perda Crítica. Segundo Fagundes et al.

O Guia para Análise Econômica de Atos de Concentração Horizontal (Portaria Conjunta SEAE/SDE nº 50, de $1^{\circ}$ de agosto de 200 I) apresenta essa sequência de análise e as variáveis consideradas. 
(2010), “a ideia da perda crítica consiste na máxima redução na quantidade vendida que o monopolista hipotético estaria disposto a incorrer para sustentar um aumento de preços" (FAGUNDES et al., 2010, p.27-28)

No Brasil, ressalta-se o estudo realizado por Ribeiro e Jacinto (2010), que usaram o teste de perda crítica para produtos diferenciados, aplicando-o no mercado de chá-pronto para beber.

Cabe ressaltar que a grande maioria dos mercados relevantes definidos pelos órgãos de defesa da concorrência em seus pareceres relativos aos Atos de Concentração utilizam uma definição qualitativa. Pode-se citar, por exemplo, a operação entre as empresas Fiat S.p.A. E Crysler LLC (Ato de Concentração 08012.003330/2009-76), cuja definição do mercado relevante na dimensão produto foi considerando a classificação feita pela Federação Nacional da Distribuição de Veículos Automotores (FENABRAVE).

Nesse sentido, o presente trabalho aborda a definição de mercado relevante por meio do Teste de Perda Crítica aplicado ao mercado automobilístico brasileiro. Pretende-se, especificamente, ver a possibilidade de formação de um único mercado relevante entre os modelos Gol, Uno e Celta no subsegmento Veículos de Entrada, por meio de simulações de aumento de preço conjunto.

\section{O TESTE DE PERDA CRÍTICA}

Segundo Fiuza (2010), o Teste do Monopolista Hipotético (TMH) foi adotado pela primeira vez pelo Departamento de Justiça (DoJ), que estava presente no Guia de análise de fusões norte-americano (Merger Guidelines) de $1982^{4}$ como metodologia de definição do mercado relevante.

O Guia norte-americano foi adotado como referência na elaboração de guias para análise de atos de concentração de vários países, inclusive o Guia brasileiro, que seguiram, dessa forma, a utilização do TMH como instrumental analítico para definição do mercado relevante ${ }^{5}$.

Segundo Massey (2000), o TMH “procura identificar o menor mercado no qual um monopolista hipotético ou cartel poderia impor um pequeno, mas significativo e não transitório aumento de preços $^{6 \%}$ (MASSEY, 2000, p.318).

Em inglês, é conhecido como SSNIP (Small, but Significant, Non-transitory, Increase in Price).

Ver o tópico sobre mercado relevante no Guia para Análise Econômica de Atos de Concentração Horizontal (Portaria Conjunta SEAE/SDE n 50, de $I^{\circ}$ de agosto de 200I).

6 Tradução livre. 
Ainda segundo Massey (2000), esse teste é feito verificando os resultados da imposição de aumento de preço de $5 \%$ por pelo menos um ano; se esse aumento não for lucrativo, ou seja, há produtos substitutos próximos ao do monopolista, então o monopolista não tem o poder de aumentar o preço. Nesse caso, o mercado relevante precisa ser expandido para incluir outros produtos ou áreas geográficas; o teste é aplicado sucessivamente até o ponto em que o monopolista hipotético puder atingir lucro com a imposição desse aumento de preço, determinado assim o conjunto de produtos e área geográfica que compõem o mercado relevante (MASSEY, 2000). Dessa forma, define-se o mercado relevante, tanto na dimensão produto como na dimensão geográfica.

Segundo O'Brien e Wickelgren (2003), desde que foi proposta, a análise do TPC tem estado presente em inúmeros casos analisados pelos órgãos antitrustes de diversos países.

De acordo com Farrell e Shapiro (2008) e Fagundes et al. (2010), o teste Perda Crítica foi introduzido por Harris e Simons (1989)7 ${ }^{7}$ O teste se baseia no cálculo da magnitude da perda de vendas (Perda Crítica - Critical Loss) que torna não lucrativo para o monopolista hipotético impor um pequeno, mas significativo e não transitório aumento de preços, e a compara com a Perda Efetiva de vendas (Actual Loss) que resultaria desse aumento de preço; se a perda real for menor do que a perda crítica, esse aumento de preço seria rentável, delineando, dessa forma, um mercado relevante (FARREL; SHAPIRO, 2008).

É importante notar que os efeitos de um aumento de preço evidenciam uma restrição básica enfrentada pelas firmas na análise antitruste: a redução na quantidade demandada. Os motivos que explicam a diminuição da quantidade demandada após um aumento de preço, pelo lado da demanda, segundo Hüschelrath (2009), podem ser explicados pelo fato de que os clientes podem reduzir seu consumo ou mesmo deixar de consumir o produto. Já pelo lado da oferta, tem que considerar as empresas rivais que produzem bens substitutos ou que podem começar a produzi-los (HÜSCHELRATH, 2009).

O cálculo do TPC pode ajudar a avaliar se o monopolista hipotético poderá usar o poder de mercado que possui para aplicar um aumento de preços pós-fusão. De acordo com Hüschelrath (2009), se a Perda Efetiva

Harris and Simons (1989). Focusing Market Definition: How Much Substitution is Necessary? Research in Law and Economics (apud FARREL; SHAPIRO, 2008) 
calculada for menor que a Perda Crítica, o monopolista hipotético (ou as firmas que realizaram a concentração) terá poder de mercado suficiente para impor um aumento de preço. Assim, quanto maior a Perda Efetiva frente à Perda Crítica, mais difícil para o monopolista hipotético estabelecer um acréscimo no preço.

De acordo com Fagundes et al. (2010), o mercado relevante estará delimitado adequadamente quando as duas medidas (Perda Crítica e Perda Efetiva) coincidirem. Se o valor de Perda Efetiva exceder o valor de Perda Crítica, o mercado relevante previamente definido deve ser ampliado; no caso contrário, deverá ser reduzido (FAGUNDES et al., 2010).

Destaca-se que, segundo Geoffroy (2011), cada forma funcional da demanda utilizada (linear, log-linear, logit e o sistema quase ideal de demanda-AIDS), implica em um nível diferente de aumento de preço. Dessa forma, a forma funcional da demanda pode influenciar o resultado do TPC. No presente trabalho, utilizam-se os valores das elasticidades no mercado de automóveis (produto diferenciado) estimados pelo modelo Mixed Logit por DeSouza, Petterini e Miro (2010) ${ }^{8}$.

\section{METODOLOGIA}

\subsection{Método do cálculo do teste de perda crítica}

O presente estudo tem como referência o trabalho de Ribeiro e Jacinto (2010), que aplicaram o teste de perda crítica para o mercado de chá-pronto para beber.

Segundo Ribeiro e Jacinto (2010), para um produto homogêneo considera-se que os produtos enfrentam os mesmos custos marginais (supostos constantes). De acordo com os autores, considera-se que $\Delta \pi \geq 0$, onde $\Delta \pi=$ $\pi 1-\pi 0$ e $\pi=q p-f-q c=q m p-f$, sendo $\pi$ é a representação dos lucros, $q$ a quantidade vendida, $f$ o custo fixo, $p$ o preço, $c$ o custo marginal constante e $m$ representa a margem preço-custo, onde $m=(p-c) / p$.

Para saber se um aumento de preço entre os períodos 0 e 1 será lucrativo, calcula-se a perda crítica (CL). De acordo com O'Brien e Wickelgren (2003), Farrel e Shapiro (2008) e Geoffroy (2011):

Mais detalhes são abordados no tópico da metodologia. 


$$
\mathrm{CL}=\mathrm{x} /(\mathrm{x}+m)
$$

onde $\mathrm{x}=\Delta \mathrm{p} / p$

Ou seja, x é a variação percentual de preço ou o SSNIP (pequeno, mas significativo e não transitório aumento de preço). Já a Perda Efetiva (PL), dada a variação de preços (x) entre os períodos 0 e 1 é calculada da seguinte forma, segundo Fagundes et al. (2010) e Geoffroy (2011):

$$
\mathrm{PL}=\mathrm{x}^{*} \varepsilon
$$

onde $\varepsilon$ representa a elasticidade-preço própria da demanda.

Segundo este teste, de acordo com Geoffroy (2011), o aumento de preços será lucrativo se a Perda Prevista (equação 2) for menor que a Perda Crítica (equação 1), ou seja, o mercado relevante deve ser mais estreito que o mercado relevante candidato ou o mercado relevante está definido. Assim, se PL > CL ou (PL/CL) > 1, o aumento de preço não será lucrativo, ou seja, a diminuição nas vendas decorrente de um aumento de preços será suficientemente grande para tornar o aumento de preços não lucrativo. Nesse caso, o mercado deve ser expandido, pois os produtos em questão não constituem um mercado relevante (o mercado relevante é definido até o ponto onde PL é igual ou semelhante à CL) (GEOFFROY, 2011).

A mesma análise deve ser feita para o produto diferenciado, entretanto, como neste caso os produtos são assimétricos em custos ou margens, a fórmula tem aspectos mais elaborados.

Dessa forma, para o cálculo do TPC para o produto diferenciado, além das elasticidade-preço da demanda próprias, novas varáveis deverão ser consideradas, a saber, as elasticidade-preço da demanda cruzadas, as margens de cada empresa e as parcelas de mercado de cada produto ${ }^{9}$ (RIBEIRO; JACINTO, 2010).

Baseando-se em Ribeiro e Jacinto (2010), que generalizaram a demonstração para dois produtos feita por Daljord (2009) ${ }^{10}$ (apud RIBEIRO;

Segundo Ribeiro e Jacinto (20 I 0), essa parcela de mercado é calculada no conjunto de empresas consideradas no teste do monopolista hipotético.

10 DALJORD, O. An exact arithmetic SSNIP teste for asymmetric products. Journal of CompetitionLaw \& Economics. 2009. 
JACINTO, 2010), considerando $i$ produtos/empresas, a variação positiva de preços será lucrativa se:

em que representa a parcela de mercado (share) nas vendas da empresa; $\Delta q i=(\varepsilon i i+\varepsilon \mathrm{ij}) * \mathrm{x}$, é a variação percentual na quantidade vendida devido ao aumento de preços (perda efetiva), onde cii é a elasticidade-preço da demanda e eij é a elasticidade cruzada; e CL $i=\mathrm{x} /(\mathrm{x}+m i)$.

Assim, para um quociente menor que um o monopolista hipotético poderá obter lucro ao implementar um SSNIP, o que define, dessa forma, o mercado relevante.

\subsection{Dados}

O mercado automobilístico brasileiro foi escolhido para a aplicação do TPC com base na disponibilidade de elasticidades já calculadas na literatu$\mathrm{ra}^{11}$, além de ser um setor com um produto diferenciado muito representativo.

Para o estudo, são utilizadas as elasticidades estimadas no trabalho de DeSouza, Petterini e Miro (2010). Para o cálculo das elasticidade-preço própria e elasticidade-preço cruzada, os autores usaram o modelo Mixed Logit para dados dos automóveis novos vendidos no Brasil entre 2005 e 2008, sendo que as elasticidades são calculadas para determinados modelos de automóveis. Também utilizam-se os markups estimados (para o ano de 2008) por DeSouza, Petterini e Miro (2010).

A base de dados para o cálculo das participações de mercado (market share) foi elaborada com as informações do Informativo mensal da Federação Nacional da Distribuição de Veículos Automotores (FENABRAVE), que apresenta dados de emplacamentos de automóveis mensalmente. Foram utilizadas as informações de emplacamentos do ano de 2013 referente ao subsegmento "Veículos de Entrada" (pertencente à categoria Automóveis), para os modelos Gol (Volkswagen - VW), Uno (Fiat) e Celta (General Motors $-\mathrm{GM})^{12}$.

"Visto que o cálculo de elasticidades não é o foco deste trabalho (e sim o cálculo do TPC), são utilizados dados de elasticidades já disponíveis na literatura.

${ }^{12}$ Considera-se, por exemplo, a família Gol (ou modelo Gol agregado), ou seja, não se consideram diferenças de 


\subsection{Mercados Relevantes Candidatos ${ }^{13}$}

De acordo com o TMH, deve-se expandir sucessivamente o mercado, a partir da definição original, até o ponto em que o monopolista hipotético puder atingir lucro. Essa expansão deve ocorrer em termos de produto ou em termos geográficos. Entretanto, para dar luz somente aos impactos da variação da "dimensão de produto", foco do presente estudo, considerou-se o nível geográfico Brasil para os mercados relevantes, conforme a definição adotada pelo Sistema Brasileiro de Defesa da Concorrência (SBDC) em seus pareceres ${ }^{14}$.

Este estudo desenvolve o teste do monopolista hipotético aplicando o cálculo do TPC para o caso "Veículos de Entrada", que é mais restrita que o segmento "Automóveis" considerando a classificação feita pela FENABRAVE. Preferiu-se escolher apenas um segmento de classificação da categoria Automóveis ${ }^{15}$ para a aplicação do TPC uma vez que essa classificação pode indicar a baixa substituição entre esses segmentos, já que os produtos são diferenciados.

São considerados os modelos mais emplacados, no subsegmento Veículos de Entrada, por cada uma das empresas Fiat, Volkswagen e General Motors. Assim, os modelos considerados no estudo são: os modelos Gol (VW), Uno (Fiat) e Celta (GM). A escolha desses modelos se deve pela representatividade desses modelos no subsegmento Veículos de Entrada, aproximadamente $65 \%$ no ano de 2013 segundo informações da FENABRAVE (2013), e também à disponibilidade de dados de elasticidades e margens dos trabalhos de DeSouza, Petterini e Miro (2010), que serão utilizadas como proxies para os modelos de carros considerados na pesquisa.

potência dos motores e de características dos modelos de Gol disponíveis.

13 Candidato se refere ao conjunto de produtos que podem (ou não) definir o mercado relevante (FAGUNDES et al., 20l0). Caso esse conjunto não delimite o mercado, é necessário agregar novos produtos/empresas para se realizar uma nova análise. Assim, cada etapa apresenta um mercado (conjunto de produtos/empresas) candidato a mercado relevante.

14 O mercado relevante nacional foi adotado, por exemplo, no Parecer SEAE n.06367/2009/RJ, referente ao Ato de Concentração n. 0801 2.003330/2009-76 entre a Fiat S.p.A e Chrysler LLC.

15 Esse subsegmento representou, em 2013, aproximadamente 29\% da categoria de Automóveis, segundo informações disponíveis no Informativo da FENABRAVE, a maior representatividade entre os demais subsegmentos. 


\subsection{Procedimento Metodológico}

Este trabalho aplica a versão mais difundida do TPC, “que supõe aumentos iguais para todas as empresas do mercado e que possui como critério uma variação não-negativa de lucros para delimitação do mercado." (RIBEIRO; JACINTO, 2010, p. 270).

Como já abordado, a metodologia do TPC é aplicada ao subsegmento de produto "Veículos de Entrada" para os principais modelos de carros no mercado geográfico brasileiro. São realizadas quatro simulações, considerando um SSNIP conjunto de 5\%. A primeira é realizada entre os modelos Uno e Gol, a segunda entre os modelos Uno e Celta, a terceira entre os modelos Gol e Celta, e por fim, a quarta (tripla) é feita entre os modelos Uno, Gol e Celta.

\subsubsection{Critérios para o uso de Elasticidades e Margens}

Para cada simulação empírica são analisados os resultados do teste para o nível de produto considerado (Veículos de Entrada) considerando um grid de margens (variável $m$ ) - de maneira análoga a Ribeiro e Jacinto (2010), que também utilizaram um grid de margens.

O grid de margem geralmente é utilizado quando há um desconhecimento das margens das empresas; com o grid será possível verificar as relações de margens que delimitam ou não o mercado relevante.

Em paralelo, também será utilizada margens de referência específica para o mercado de automóveis, calculadas em DeSouza, Petterini e Miro $(2010)^{16}$. Essa margem permite verificar com maior precisão se delimita ou não o mercado relevante, já que se refere a uma margem estimada, e portanto, conhecida.

DeSouza, Petterini e Miro (2010) também encontraram índices de elasticidades para diversos tipos de modelos de automóveis. Entretanto, como este estudo considera apenas modelos agregados (modelo Gol, por exemplo) nas simulações, consideram-se as elasticidades e os markups dos modelos Gol 1.0, Celta 1.0 e Uno Mille estimados no trabalho de DeSouza, Petterini e Miro (2010) como proxies.

\footnotetext{
${ }^{6}$ Markups estimados.
} 
Assim, o trabalho utilizou tanto o grid de margem como a margem de referência, apresentando ambos os resultados obtidos para a delimitação do mercado relevante.

Ressalta-se que as simulações de um aumento de preço conjunto entre modelos de automóveis, considerando tanto o grid de margem como a margem de referência, são feitas para margens simétricas e assimétricas. Para as simulações triplas (entre três modelos de carros) são representados os critérios para a delimitação do mercado relevante para um grid de margens somente simétrica, pois a representação do critério $\left(\sum_{i} s_{i} \Delta q_{i} / C L_{i}\right)$ fica muito complexa para as margens assimétricas.

\subsection{Limitações da Aplicação do Teste}

A primeira limitação se refere à consideração de análise de mercado relevante para o segmento de produtos Veículos de Entrada, que por ser mais restrito, pode levar a uma superestimação do poder de mercado das empresas.

Uma segunda limitação é o uso de proxies de elasticidades e markups para os modelos considerados no presente estudo: Gol (ou família Gol), Uno (ou família Uno) e Celta (ou família Celta).

Por fim, outra limitação se refere à diferença temporal entre os dados de vendas de automóveis - FENABRAVE (2013) - e as elasticidades de DeSouza, Petterini e Miro (2010), para cujos cálculos foram utilizados dados de 2005 a 2008. Assim, o presente estudo considera que os índices de elasticidades não se alteraram entre 2005-2008 e 2013.

\section{RESULTADOS}

A Tabela 1 apresenta os valores de Perda Crítica enfrentados para um grid de margens com a aplicação de um SSNIP de 5\%. Nota-se que as variáveis são inversamente proporcionais; ou seja, quanto maior a margem da empresa, menor o índice de perda crítica. 
Tabela 1 - Grid de Margens e Perda Crítica

\begin{tabular}{lllllllllll}
\hline Margem & $5 \%$ & $10 \%$ & $15 \%$ & $20 \%$ & $25 \%$ & $30 \%$ & $35 \%$ & $40 \%$ & $45 \%$ & $50 \%$ \\
\hline Perda & & & & & & & & & & \\
Crítica & $-50,0 \%$ & $-33,3 \%$ & $-25,0 \%$ & $-20,0 \%$ & $-16,7 \%$ & $-14,3 \%$ & $-12,5 \%$ & $-11,1 \%$ & $-10,0 \%$ & $-9,1 \%$ \\
(CL) & & & & & & & & & & \\
\hline
\end{tabular}

Fonte: elaborado pelos autores.

A seguir, apresentam-se as simulações entre os modelos de carros para verificar se formam um mercado relevante conjunto. Para isso, considera-se um SSNIP, conjunto, de 5\%, simultaneamente.

\section{A) Uno e Gol}

Aplicam-se as elasticidades mensuradas pelo modelo Mixed Logit por DeSouza, Petterini e Miro (2010) aos modelos de automóvel Uno Mille e Gol 1.0. Utilizam-se as elasticidades e margens desses modelos de automóveis, visto que são os modelos mais vendidos pelas firmas no subsegmento de produto Veículos de Entrada.

As elasticidades para Uno Mille e Gol 1.0 mensurados por DeSouza, Petterini e Miro (2010) estão na Tabela 2.

Tabela 2 - Elasticidades-preço utilizadas: Uno Mille e Gol 1.0

\begin{tabular}{l|l|l|l}
\hline \multirow{2}{*}{ Variação na demanda } & \multicolumn{3}{|c}{ Variação no preço } \\
\cline { 2 - 4 } & & Uno Mille & Gol 1.0 \\
\cline { 2 - 4 } & Uno Mille & $-5,87$ & 0,52 \\
\cline { 2 - 4 } & Gol 1.0 & 1,10 & $-4,01$ \\
\hline
\end{tabular}

Fonte: com base em DeSouza, Petterini e Miro (2010).

A Tabela 3 apresenta os resultados dos critérios de determinação do mercado relevante $\left(\sum_{i} s_{i} \Delta q_{i} / C L_{i}\right)$, considerando um grid de margens, a aplicação de um SSNIP conjunto de 5\% (ou seja, ambos os modelos tiveram um aumento de preço de 5\% simultaneamente), as parcelas de mercado (shares) de 0,42 (ou $42 \%$ ) para o Uno e 0,58 (ou 58\%) para o Gol (considerando o mercado candidato apenas para esses modelos), e as elasticidades apresentadas na Tabela 2.

Os resultados mostram que para margens simétricas - diagonal principal - as empresas da simulação hipotética conterão um mercado relevante 
conjunto para o nível de produto Veículos de Entrada se as margens forem iguais ou menores que 0,20 . No caso de margens assimétricas, nota-se que as margens do modelo Gol podem ser maiores que a do Uno e ainda assim determinar o mercado relevante.

Tabela 3 - Critérios para Determinação do Mercado Relevante para Veículos de Entrada: Gol e Uno

Gol

\begin{tabular}{|c|c|c|c|c|c|c|c|c|c|c|}
\hline Margens & 0,05 & 0,10 & 0,15 & 0,20 & 0,25 & 0,30 & 0,35 & 0,40 & 0,45 & 0,50 \\
\hline 0,05 & 0,39 & 0,48 & 0,56 & 0,65 & 0,73 & 0,82 & 0,90 & 0,98 & 1,07 & 1,15 \\
\hline 0,10 & 0,51 & 0,59 & 0,67 & 0,76 & 0,84 & 0,93 & 1,01 & 1,10 & 1,18 & 1,27 \\
\hline 0,15 & 0,62 & 0,70 & 0,79 & 0,87 & 0,96 & 1,04 & 1,12 & 1,21 & 1,29 & 1,38 \\
\hline 0,20 & 0,73 & 0,81 & 0,90 & 0,98 & 1,07 & 1,15 & 1,24 & 1,32 & 1,41 & 1,49 \\
\hline 0,25 & 0,84 & 0,93 & 1,01 & 1,10 & 1,18 & 1,26 & 1,35 & 1,43 & 1,52 & 1,60 \\
\hline 0,30 & 0,95 & 1,04 & 1,12 & 1,21 & 1,29 & 1,38 & 1,46 & 1,55 & 1,63 & 1,71 \\
\hline & 1,07 & 1,15 & 1,24 & 1,32 & 1,40 & 1,49 & 1,57 & 1,66 & 1,74 & 1,83 \\
\hline 0,40 & 1,18 & 1,26 & 1,35 & 1,43 & 1,52 & 1,60 & 1,69 & 1,77 & 1,85 & 1,94 \\
\hline 0,45 & 1,29 & 1,38 & 1,46 & 1,54 & 1,63 & 1,71 & 1,80 & 1,88 & 1,97 & 2,05 \\
\hline 0,50 & 1,40 & 1,49 & 1,57 & 1,66 & 1,74 & 1,83 & 1,91 & 1,99 & 2,08 & 2,16 \\
\hline
\end{tabular}

Fonte: elaborado pelos autores.

Nota: os critérios em negrito indicam as relações de margens que tornam o mercado relevante definido.

Agora avalia-se o resultado do critério $\left(\sum_{i} s_{i} \Delta q_{i} / C L_{i}\right)$ tomando como referência a margem estimada no estudo de DeSouza, Petterini e Miro (2010) para os modelos de carros em questão ${ }^{17}$. O critério obtido com essas margens de referência é de aproximadamente 0,92. Considerando essas margens de referência, os modelos Uno e Gol, para o nível de produto Veículos de Entrada, representam um mercado relevante conjunto. Isso implica que é lucrativo para esse conjunto de empresas (Fiat e VW) impor um SSNIP de 5\% para os modelos Uno e Gol no mercado de Veículos de Entrada. Dessa forma não há necessidade de agregar novos modelos de carros, como por exemplo o Celta, ao mercado relevante candidato e fazer um novo teste.

17 As margens de referências utilizadas são os markups estimados no estudo de DeSouza, Petterini e Miro (20 I 0): Uno Mille aproximadamente 0, 17 (ou 17\%) e Gol I.0 aproximadamente 0,20 (ou 20\%). Dada essas margens de referência, percebe-se que as margens mais próximas encontradas na Tabela 3 são: 0,20 para o Gol e 0, I5 para o Uno (cruzando essas margens nesta tabela obtém-se o resultado do critério 0,87 ). 


\section{B) Uno e Celta}

Aplica-se as elasticidades mensuradas por DeSouza, Petterini e Miro (2010) aos modelos de automóvel Uno Mille e Celta 1.0, dispostas na Tabela 4 .

TABELA 4 - Elasticidades-preço utilizadas: Uno Mille e Celta 1.0

\begin{tabular}{l|l|l|l}
\hline & \multicolumn{3}{|c}{ Variação no preço } \\
\hline \multirow{2}{*}{ Variação na demanda } & & Uno Mille & Celta 1.0 \\
\cline { 2 - 4 } & Uno Mille & $-5,87$ & 0,91 \\
\cline { 2 - 4 } & Celta 1.0 & 1,10 & $-5,08$ \\
\hline
\end{tabular}

Fonte: com base em DeSouza, Petterini e Miro (2010).

Os critérios $\left(\sum_{i} s_{i} \Delta q_{i} / C L_{i}\right)$ da tabela 5 foram calculados considerando um SSNIP, conjunto, de 5\%, parcelas de mercado de 0,71 para o Uno e 0,29 para o Celta (considerando apenas esses modelos no mercado candidato), as elasticidades da Tabela 4 e um grid de margens.

TABELA 5 - Critérios para determinação do mercado relevante para Veículos de Entrada: Uno e Celta

Celta

\begin{tabular}{l|lllllllllll}
\hline Margens & 0,05 & 0,10 & 0,15 & 0,20 & 0,25 & 0,30 & 0,35 & 0,40 & 0,45 & 0,50 & 0,55 \\
\hline 0,05 & $\mathbf{0 , 4 7}$ & $\mathbf{0 , 5 3}$ & $\mathbf{0 , 5 8}$ & $\mathbf{0 , 6 4}$ & $\mathbf{0 , 7 0}$ & $\mathbf{0 , 7 5}$ & $\mathbf{0 , 8 1}$ & $\mathbf{0 , 8 7}$ & $\mathbf{0 , 9 3}$ & $\mathbf{0 , 9 8}$ & 1,04 \\
0,10 & $\mathbf{0 , 6 4}$ & $\mathbf{0 , 7 0}$ & $\mathbf{0 , 7 6}$ & $\mathbf{0 , 8 2}$ & $\mathbf{0 , 8 7}$ & $\mathbf{0 , 9 3}$ & $\mathbf{0 , 9 9}$ & 1,05 & 1,10 & 1,16 & 1,22 \\
0,15 & $\mathbf{0 , 8 2}$ & $\mathbf{0 , 8 8}$ & $\mathbf{0 , 9 4}$ & $\mathbf{0 , 9 9}$ & 1,05 & 1,11 & 1,16 & 1,22 & 1,28 & 1,34 & 1,39 \\
0,20 & $\mathbf{1 , 0 0}$ & 1,05 & 1,11 & 1,17 & 1,23 & 1,28 & 1,34 & 1,40 & 1,46 & 1,51 & 1,57 \\
$\stackrel{5}{5} 0,25$ & 1,17 & 1,23 & 1,29 & 1,35 & 1,40 & 1,46 & 1,52 & 1,58 & 1,63 & 1,69 & 1,75 \\
0,30 & 1,35 & 1,41 & 1,47 & 1,52 & 1,58 & 1,64 & 1,69 & 1,75 & 1,81 & 1,87 & 1,92 \\
0,35 & 1,53 & 1,58 & 1,64 & 1,70 & 1,76 & 1,81 & 1,87 & 1,93 & 1,99 & 2,04 & 2,10 \\
0,40 & 1,70 & 1,76 & 1,82 & 1,88 & 1,93 & 1,99 & 2,05 & 2,10 & 2,16 & 2,22 & 2,28 \\
0,45 & 1,88 & 1,94 & 1,99 & 2,05 & 2,11 & 2,17 & 2,22 & 2,28 & 2,34 & 2,40 & 2,45 \\
0,50 & 2,06 & 2,11 & 2,17 & 2,23 & 2,29 & 2,34 & 2,40 & 2,46 & 2,52 & 2,57 & 2,63 \\
0,55 & 2,23 & 2,29 & 2,35 & 2,41 & 2,46 & 2,52 & 2,58 & 2,63 & 2,69 & 2,75 & 2,81 \\
\hline
\end{tabular}

Fonte: elaborado pelos autores.

Nota: os critérios em negrito indicam as relações de margens que tornam o mercado relevante definido. 
Os resultados mostram que para margens simétricas os modelos formam um mercado relevante conjunto para o nível de produto Veículos de Entrada se as margens forem iguais ou menores que 0,15. Já para as margens assimétricas, nota-se que as margens do modelo Celta podem ser maiores que o do Uno e ainda assim determinar o mercado relevante.

Em relação às margens de referência ${ }^{18}$ chegou-se ao seguinte critério: 1,03 , valor muito próximo da fronteira de delimitação do mercado relevante, mas que ainda não torna não lucrativo para o conjunto de empresas um SSNIP.

Assim, é necessário agregar um novo modelo de automóvel do subsegmento de produto Veículos de Entrada, como por exemplo o Gol, ao mercado relevante candidato para se executar um novo TPC.

\section{C) Gol e Celta}

A Tabela 6 apresenta os valores das elasticidades mensuradas no estudo de DeSouza, Petterini e Miro (2010) para os modelos de automóvel Gol 1.0 e Celta 1.0 .

TABELA 6 - Elasticidades-preço utilizadas: Gol 1.0 e Celta 1.0

\begin{tabular}{l|l|l|l}
\hline & \multicolumn{3}{|c}{ Variação no preço } \\
\hline \multirow{2}{*}{ Variação na demanda } & & Gol 1.0 & Celta 1.0 \\
\cline { 2 - 4 } & Gol 1.0 & $-4,01$ & 0,84 \\
\cline { 2 - 4 } & Celta 1.0 & 0,48 & $-5,08$ \\
\hline
\end{tabular}

Fonte: com base em DeSouza, Petterini e Miro (2010).

Os resultados $\left(\sum_{i} s_{i} \Delta q_{i} / C L_{i}\right)$ para o nível de produto Veículos de Entrada estão na Tabela 7, e foram calculados considerando a aplicação de um SSNIP conjunto de $5 \%$ e parcelas de mercado de 0,23 para o Celta e 0,77 para o Gol (considerando o mercado candidato apenas para esses modelos), as elasticidades da tabela 6 e um grid de margens.

Verifica-se que para margens simétricas, o mercado relevante é definido para margens iguais ou inferiores a 0,20 . No caso de margens assimétricas, nota-se que as margens do modelo Celta podem ser maiores que o do Gol e ainda assim determinar o mercado relevante.

18 Margens de referência do trabalho de DeSouza, Petterini e Miro (20 I 0): Uno Mille aproximadamente 0, I7 e Celta 1.0 aproximadamente 0,17 . 
TABELA 7 - Critérios para determinação do mercado relevante para Veículos de Entrada: Gol e Celta

\begin{tabular}{l|rrrrrrrrrrrrrr}
\multicolumn{10}{c}{ Celta } \\
\hline Margens & 0,05 & 0,10 & 0,15 & 0,20 & 0,25 & 0,30 & 0,35 & 0,40 & 0,45 & 0,5 & 0,55 & 0,6 & 0,65 & 0,7 \\
\hline 0,05 & $\mathbf{0 , 3 5}$ & $\mathbf{0 , 4 0}$ & $\mathbf{0 , 4 5}$ & $\mathbf{0 , 5 1}$ & $\mathbf{0 , 5 6}$ & $\mathbf{0 , 6 1}$ & $\mathbf{0 , 6 6}$ & $\mathbf{0 , 7 1}$ & $\mathbf{0 , 7 7}$ & $\mathbf{0 , 8 2}$ & $\mathbf{0 , 8 7}$ & $\mathbf{0 , 9 2}$ & $\mathbf{0 , 9 7}$ & 1,03 \\
0,10 & $\mathbf{0 , 4 7}$ & $\mathbf{0 , 5 2}$ & $\mathbf{0 , 5 8}$ & $\mathbf{0 , 6 3}$ & $\mathbf{0 , 6 8}$ & $\mathbf{0 , 7 3}$ & $\mathbf{0 , 7 8}$ & $\mathbf{0 , 8 4}$ & $\mathbf{0 , 8 9}$ & $\mathbf{0 , 9 4}$ & $\mathbf{0 , 9 9}$ & 1,04 & 1,10 & 1,15 \\
0,15 & $\mathbf{0 , 5 9}$ & $\mathbf{0 , 6 5}$ & $\mathbf{0 , 7 0}$ & $\mathbf{0 , 7 5}$ & $\mathbf{0 , 8 0}$ & $\mathbf{0 , 8 5}$ & $\mathbf{0 , 9 1}$ & $\mathbf{0 , 9 6}$ & 1,01 & 1,06 & 1,12 & 1,17 & 1,22 & 1,27 \\
0,20 & $\mathbf{0 , 7 2}$ & $\mathbf{0 , 7 7}$ & $\mathbf{0 , 8 2}$ & $\mathbf{0 , 8 7}$ & $\mathbf{0 , 9 3}$ & $\mathbf{0 , 9 8}$ & 1,03 & 1,08 & 1,13 & 1,19 & 1,24 & 1,29 & 1,34 & 1,39 \\
$\overline{0}$ & $\mathbf{0 , 8 4}$ & $\mathbf{0 , 8 9}$ & $\mathbf{0 , 9 4}$ & $\mathbf{1 , 0 0}$ & 1,05 & 1,10 & 1,15 & 1,20 & 1,26 & 1,31 & 1,36 & 1,41 & 1,46 & 1,52 \\
0,25 & $\mathbf{0 , 9 6}$ & 1,01 & 1,07 & 1,12 & 1,17 & 1,22 & 1,27 & 1,33 & 1,38 & 1,43 & 1,48 & 1,54 & 1,59 & 1,64 \\
0,30 & 1,09 & 1,14 & 1,19 & 1,24 & 1,29 & 1,35 & 1,40 & 1,45 & 1,50 & 1,55 & 1,61 & 1,66 & 1,71 & 1,76 \\
0,35 & 1,21 & 1,26 & 1,31 & 1,36 & 1,42 & 1,47 & 1,52 & 1,57 & 1,62 & 1,68 & 1,73 & 1,78 & 1,83 & 1,88 \\
0,40 & 1,33 & 1,38 & 1,43 & 1,49 & 1,54 & 1,59 & 1,64 & 1,69 & 1,75 & 1,80 & 1,85 & 1,90 & 1,96 & 2,01 \\
0,45 & 1,45 & 1,50 & 1,56 & 1,61 & 1,66 & 1,71 & 1,77 & 1,82 & 1,87 & 1,92 & 1,97 & 2,03 & 2,08 & 2,13 \\
0,50 & & & & &
\end{tabular}

Fonte: elaborado pelos autores.

Nota: os critérios em negrito indicam as relações de margens que tornam o mercado relevante definido.

Agora considerando as margens de referência ${ }^{19} \mathrm{o}$ mercado relevante é definido para esses dois modelos de carros, pois o critério foi de 0,84. Assim, é lucrativo para esse conjunto de modelos de carros impor um SSNIP de 5\% no mercado de Veículos de Entrada. Dessa forma não há necessidade de agregar novos modelos de carros (como por exemplo o Uno) ao mercado relevante candidato e fazer um novo teste.

\section{D) Uno, Gol e Celta}

Considerando uma simulação tripla de modelos de carros, ou seja, quando as empresas Fiat, GM e VW (respectivamente para os modelos Uno, Celta e Gol) aumentam, em conjunto, os preços em 5\% simultaneamente.

Verificam-se os resultados encontrados com a utilização das elasticidades calculados no estudo de DeSouza, Petterini e Miro (2010). Os resultados para o grid de margens simétricas com a aplicação de um SSNIP estão na Tabela 8 .

${ }_{19}$ Margens de referência do trabalho de DeSouza, Petterini e Miro (20।0): Gol I.0 aproximadamente 0,20 e Celta I.0 aproximadamente 0,17 . 
TABELA 8 - Critérios para determinação do mercado relevante para Veículos de Entrada: Uno, Gol e Celta

\begin{tabular}{|c|c|}
\hline Margens & Critério \\
\hline 0,05 & $\mathbf{0 , 3 1}$ \\
\hline 0,1 & $\mathbf{0 , 4 7}$ \\
\hline 0,15 & $\mathbf{0 , 6 2}$ \\
\hline 0,2 & $\mathbf{0 , 7 8}$ \\
\hline 0,25 & $\mathbf{0 , 9 3}$ \\
\hline 0,3 & 1,09 \\
\hline 0,35 & 1,24 \\
\hline 0,4 & 1,40 \\
\hline 0,45 & 1,55 \\
\hline 0,5 & 1,71 \\
\hline
\end{tabular}

Fonte: elaborado pelos autores.

Nota: emprega-se shares de 0,36 para o Uno, 0,50 para o Gol e 0,14 para o Celta.

Assim, para as margens simétricas inferiores ou iguais a 0,25 o mercado relevante é delimitado para esse grupo de modelos de carros (Gol, Uno e Celta) no nível de produto Veículos de Entrada, pois é possível obter lucro com a imposição conjunta de um SSNIP de 5\%.

Considerando as margens de referência, o critério obtido foi de 0,71. Portanto, esses três modelos de carros poderiam constituir um mercado relevante.

\section{CONSIDERAÇÕES FINAIS}

Realizou-se um estudo aplicando o TPC para a definição de mercado relevante, seguindo a lógica pregada pela TMH. Assim, foi possível examinar os resultados do TPC para algumas simulações entre modelos de carros para o caso de Veículos de Entrada no mercado brasileiro, levando-se em conta um SSNIP conjunto de 5\%. Os modelos considerados no trabalho foram os modelos Gol, Uno e Celta.

Ressalta-se que devido à falta de dados disponíveis para o segmento Veículos de Entrada, cada um destes modelos foi tomado de forma agregada, 
ou seja, sem considerar as diferenças de potência do motor ou características do carro. Ademais, proxies para a elasticidade e markups foram utilizadas.

Observou-se que a delimitação do mercado relevante fica dependente da margem considerada, o que foi possível verificar através dos resultados encontrados para o grid de margem e para as margens de referência.

Na primeira simulação entre os modelos Uno e Gol verificou-se que, para o nível de produto Veículos de Entrada, esses modelos formariam um mercado relevante conjunto para as margens simétricas iguais ou menores que 0,20 . Já em relação às margens assimétricas, as margens do modelo Gol podem ser maiores que o do Uno e ainda assim determinar o mercado relevante. No caso da utilização dos markups (margens de referência) estimados por DeSouza, Petterini e Miro (2010), os modelos Uno e Gol, para o nível de produto Veículos de Entrada, representam um mercado relevante conjunto.

Já para a segunda simulação realizada, entre os modelos Celta e Uno, verificou-se que para as margens simétricas os modelos formam um mercado relevante conjunto para o nível de produto Veículos de Entrada se essas margens forem iguais ou menores que 0,15 . Já para as margens assimétricas, nota-se que as margens do modelo Celta podem ser maiores que o do Uno e ainda assim determinar o mercado relevante. Observou-se que esses modelos não formariam um mercado relevante conjunto quando se consideram as margens de referência, ou seja, outros modelos deveriam ser incorporados ao mercado relevante.

$\mathrm{Na}$ terceira simulação (entre os modelos Celta e Gol) realizada, observou-se que para as margens simétricas o mercado relevante é definido para margens iguais ou inferiores a 0,20. Já para as margens assimétricas, os resultados indicaram que as margens do modelo Celta podem ser maiores que o do Gol e ainda assim determinar o mercado relevante. Ao considerar as margens de referência, o mercado relevante é definido para esses dois modelos de carros.

Por fim, em relação à simulação tripla - entre os modelos de carros Uno, Gol e Celta - verificou-se que para as margens simétricas inferiores ou iguais a 0,25 o mercado relevante é delimitado para esses modelos. Já considerando as margens de referência, o mercado relevante é definido para esses três modelos. 
Observa-se nas simulações, considerando as margens de referência, que o modelo Gol 1.0 é forte candidato de estar no mesmo mercado relevante que o Celta 1.0 e o Uno 1.0.

Dados os resultados obtidos utilizando as margens de referência na terceira simulação, quando os três modelos de carros têm um SSNIP conjunto de $5 \%$ pode-se dizer que a opção em considerar a definição de mercado utilizando a segmentação da FENABRAVE como Veículos de Entrada é uma alternativa.

Nesse sentido, a proposta desse trabalho foi aplicar o TPC como um método auxiliar na definição de mercado relevante, ou seja, ao avaliar a possibilidade de exercício de poder de mercado.

\section{CRITICAL LOSS ANALYSIS FOR THE RELEVANT MARKET DELINEATION: A SIMULATION FOR THE AUTOMOTIVE MARKET}

\section{Abstract}

The relevant market definition is important for merger analysis in the antitrust policy, according to the Hypothetical Monopolist Test (TMH). The critical loss test is used to measure the relevant market to achieve this goal. Thus, this paper apply the Critical Loss Test for the Brazilian car market, specifically for three models (Uno, Gol and Celta), in the subsegment Entrance Vehicle. The main result showed that the definition of the relevant market on the simulations depends on the margin considered. It is observed in the simulations, considering the reference margins, the Gol 1.0 model is a possible candidate to be in the same relevant market as the Celta 1.0 and the Uno 1.0.

Keywords: Relevant market; Critical loss analysis; Market power, Automobile; Entrance vehicle

JEL Classification: L1, L4 


\section{REFERÊNCIAS}

BRASIL. Portaria n ${ }^{\circ} 50$ da SEAE/SDE, de 1 de Agosto de 2001. Disponível em: www.seae.gov.br. Acesso em: 28 maio de 2012.

BRASIL. Conselho Administrativo de Defesa Econômica. Ato de Concentração $n^{\circ}$ 08012.003330/2009-76. Disponível em: www.cade.gov.br. Acesso em: 14 dez. 2014.

DALJORD, O. An exact arithmetic SSNIP teste for asymmetric products. Journal of Competition Law \& Economics. 2009.

DESOUZA, S. A.; PETTERINI, F. C., MIRO, V. H. A tributação nas vendas de automóveis no Brasil: quem paga a maior parte da conta? Revista EconomiA. Brasília, v.11, n.3, p.559-596. 2010.

FAGUNDES, J.; NISHIJIMA, M; LIMA, M. A. M.; ROCHA, M. M.; LAGROTERIA, E. Sobre a determinação de mercados relevantes: teste de elasticidade crítica e teste de perda crítica. Suplemento eletrônico da Revista do IBRAC, ano 1, n. 2, p.26-32, abril de 2010.

FARRELL, J.; SHAPIRO, C. Improving critical loss analysis. The Antitrust Source. 2008. Disponível em: http:/faculty.haas.berkeley.edu/shapiro/ critical2008.pdf. Acesso em: 15 abr. 2013.

FENABRAVE - Federação Nacional da Distribuição de Veículos Automotores. Índices e Números - Resumo Mensal Dez/2013. Disponível em: http:// www3.FENABRAVE.org.br:8082/plus/. 2013. Acesso em: 15 nov. 2014.

FIUZA, E. P. S. Delimitação de mercado relevante e simulação de fusões horizontais: uma abordagem unificada. Texto para discussão $n^{\circ} 1467$. Instituto de Pesquisa Econômica Aplicada (IPEA). Rio de Janeiro, janeiro de 2010.

GEOFFROY, R.C. O Novo Horizontal Merger Guidelines e os Desenvolvimentos Recentes nas Metodologias de Delimitação do Mercado Relevante. Prêmio SEAE. p.1-59. 2011. 
HARRIS, B; SIMONS, J. Focusing Market Definition: How Much Substitution is Necessary? Research in Law and Economics. 1989.

HÜSCHELRATH, K. Critical Loss Analysis in Market Definition and Merger Control. ZEW - Centre for European Economic Research. Texto para discussão no. 09-083, p.1-31. 2009.

MASSEY, P. Market Definition and Market Power in Competition Analysis: Some Practical Issues. The Economic and Social Review, vol.31, n.4, p.309-328. 2000.

O'BRIEN, D. P.; WICKELGREN, A. L. A Critical Analysis of Critical Loss Analysis. Federal Trade Commission - working paper no 254, p.1-31. 2003.

POSSAS, M. L. Os conceitos de mercado relevante e de poder de mercado no âmbito da defesa da concorrência. Revista do IBRAC, v.1, p.1-19.1996.

RIBEIRO, E. P.; JACINTO, P. A. Delimitação de mercado relevante para produtos diferenciados usando elasticidades: o caso de chá pronto para beber. Revista EconomiA, (Selecta). Brasília, vol. 11(4), p. 267-284. 2010. 